\title{
Mechanical behavior study of laminate composite by three-color digital holography
}

\author{
M Karray ${ }^{1,2^{*}}$, C Poilane $^{3}$, M Gargouri $^{1}$ and P Picart ${ }^{2}$
}

\begin{abstract}
A method for real time 3D measurements based on three-color digital holographic interferometry is presented and applied to the investigation of fracture mechanisms in laminate composite submitted to a three point flexural loading. A convolution algorithm allows the three monochrome images to be superposed to provide simultaneous full-field 3D measurements. Experimental results are presented and exploited to obtain the evolution of the crack tip propagation during the test.
\end{abstract}

Keywords: Laminate composite, Three-color digital holography, Three point flexural loading, Displacement measurement

\section{Background}

Presently, composite materials are used very efficiently in structures aeronautics, automobile transport, and the shipbuilding or in the building clearly increased during these last ten year. Therefore, it is necessary to have a better understanding of the influence of damage on the mechanical behaviour of composite materials. Different methods are used to measure surface displacements, crack propagation or simply detect the presence of defects. Y. Y. Hung and W. Steinchen used a Shearographic nondestructive testing relies on measuring the reponse of a defect to stresses, in particular, of laminated composite structures [1-3]. Others authors developed methods based on speckle interferometry (SLBI), which are efficiently used in several interferometriy techniques as an information carrier of the macroscopic wave front distortion induced by the surface displacement field of the object under investigation [4-6]. Therefore some of these methods have limitations such as scale and segmentation and they deliver data that are abundant but partial. Furthermore, such an investigation needs a multidimensional deformation measurement.

\footnotetext{
* Correspondence: mayssakarray@gmail.com

'Unité de l'état solide, Faculté des Sciences de Sfax, Route Soukra, 3018 Sfax, Tunisie

${ }^{2}$ LAUM UMR CNRS 6613, Université du Maine, Av. O. Messiaen, 72085 Le

Mans, France

Full list of author information is available at the end of the article
}

Digital holography has become properly available since its confirmation was established in the 90's [7, 8]. Theory and reconstruction algorithms for digital holography, according to the different possible schemes for the recording, have been described by several authors [8]. Particularly, holographic techniques give a fruitful contribution to the analysis of mechanical structures under strain, by providing whole field information on displacement $[9,10]$.

Digital color holography provides therefore a very interesting opportunity for the simultaneous multidimensional deformation measurement [11-14].

In this way, we present in this paper a method of realtime 3D deformation measurements based on three-color digital holography and simultaneous recording with a stack of photodiodes image sensor. The method results in a 3D measurement of the deformations of laminate composite submitted to a three point flexural loading.

\section{Experimental details \\ Materials}

The materials used in this work are glass/epoxy unidirectional laminates. Our test specimen is a rectangular plate following proportioning: fiber 50\%, epoxy resin $37.5 \%$ and $12.5 \%$ of hardener. The "Technique of Elaboration" consists of applying successively into a mould surface, a layer of resin (epoxy) a layer of reinforcement (glass fiber) and to impregnate the reinforcement by hand with the aid of a roller [15]. The stacking sequence consisted of 12 layers. 
Polymerization is achieved at room temperature during approximately $12 \mathrm{~h}$ period under pressure. After that, this plate is cut using a diamond wheel saw. The specimens obtained are $95 \mathrm{~mm}$ in length, and $30 \mathrm{~mm}$ in width with $4 \mathrm{~mm}$ of thickness. The type of samples is a $0^{\circ}$ off-axis unidirectional. To evaluate the influence of the size of the body incorporated on the mechanical behavior of material, one metal patch in the form of disc is integrated within each test-beam, so we obtained tow test specimens: one simple laminate beam (SLB) and other incorporated laminate beam (ILB) with patch.

\section{Optical set-up}

The experimental set-up adapted to the investigation of the three point flexural loading, is described in Fig. 1. It uses three continuous diode-pumped solid-state lasers (red line $\mathrm{R}$ at $\lambda_{R}=671 \mathrm{~nm}$, green line $\mathrm{G}$ at $\lambda_{G}=532 \mathrm{~nm}$, and blue line $B$ at $\lambda_{B}=457 \mathrm{~nm}$ ). The color sensor consists of a color camera made up of three stacked layers of photodiodes with a single 8 bit per channel digital output, including $(\mathrm{M}, \mathrm{N})=(1060,1420)$ pixels with pitches $p_{x}=p_{y}=5 \mu \mathrm{m}$. Each collimated laser beam illuminates the object under interest with $\theta_{R}, \theta_{G}$, and $\theta_{B}$ angles, respectively for the red, green, and blue lines leading to the three sensitivities. The $\mathrm{R}$ and $\mathrm{G}$ beams are included in the $\{x, z\}$ plane, whereas the B beam is included in the $\{y, z\}$ plane. The three beams are separated in reference and object beams by cubes PBS1, PBS2 and PBS3. The R and B reference beams are combined into an unique beam thanks to the use of a dichroic plate, which it reflects the $\mathrm{B}$ beam and transmits the $\mathrm{R}$ beam, while $\mathrm{G}$ is adjusted independently of the two other beams.

The smooth plane reference wave $G$ is produced through the SF1, which includes an achromatic lens although the RB reference waves are, produced the SF2. Thus, the two reference beams RB impact the sensing area with the same incidence angle to produce spatial frequencies $\left(u_{R}=-28.54 ; v_{R}=-51.34\right) \mathrm{mm}^{-1}$ of the red hologram and $\left(u_{B}=-41.91 ; v_{B}=-75.39\right) \mathrm{mm}^{-1}$ of the Blue hologram. The green reference beam is regulated with an incidence angle leading to spatial frequencies $\left(u_{G}=45.82\right.$; $\left.v_{G}=-30.73\right) \mathrm{mm}^{-1}$ of green hologram.

The reconstructing horizon is chosen equal $\mathrm{K} \times \mathrm{L}=1024 \times 1024$ data points.

The reconstruction algorithm follows the method based on convolution method with an adjustable magnification [16]. The method is based on the image locations and magnification relations of holography when the illuminating beam is a spherical wave front. The curvature radius of the spherical wave front is $R_{c}$ with the transversal magnification between the reconstructed object and the real one is given by:

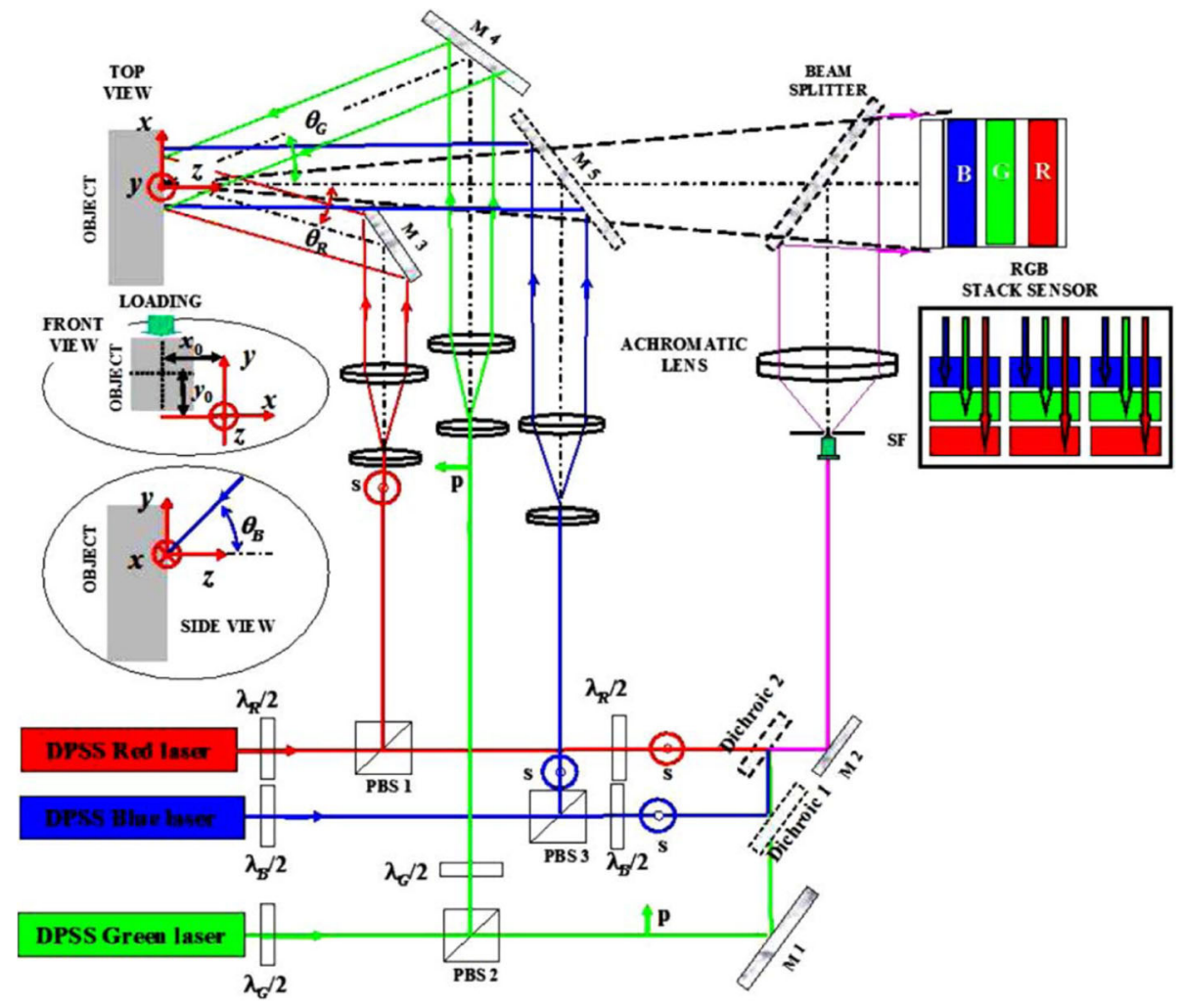

Fig. 1 Experimental setup 


$$
\gamma=-\frac{d_{r}}{d_{0}}
$$

Thus the reconstruction distance $d_{r}$ depends on $R_{c}$ and the curvature of the spherical wave, as shown in this relation:

$$
d_{r}=-\left(\frac{1}{d_{0}}+\frac{1}{R_{c}}+\frac{1}{d_{s}}\right)^{-1}
$$

The transversal magnification and the number of points of the algorithm are linked by this expression.

$$
\{K, L\}=|\gamma|\left\{\frac{\Delta A_{x}}{p_{x}}, \frac{\Delta A_{x}}{p_{x}}\right\}
$$

According to the potentialities of this strategy, the transverse magnification that must be put into the algorithm is $\gamma=0.16$, the curvature radius of the numerical spherical reconstructing wave is $R_{c}=\gamma d_{0} /$ $(\gamma-1)=-157.3481 \mathrm{~mm}$; and the effective reconstruction distance is $d_{r}=-\gamma d_{0}=-132.27 \mathrm{~mm}$, for each wavelength.

The object is illuminated from three directions, which produces three sensitivity vectors and, subsequently, a three-sensitivity measurement. The relation between the displacement vector $\mathbf{U}=u_{x} \mathbf{i}+u_{y} \mathbf{j}+u_{z} \mathbf{k}$ and the illuminating geometry is $\Delta \phi_{\lambda}=2 \pi \mathbf{U} .\left(\mathbf{K}_{e}^{\lambda}-\mathbf{K}_{\mathbf{o}}\right) / \lambda$ for each wavelength, where $\mathbf{K}_{e}^{\lambda}=-\cos \theta_{y z}^{\lambda} \sin \theta_{x z}^{\lambda} \mathbf{i}-\sin \theta_{y z}^{\lambda} \mathbf{j}-\cos \theta_{y z}^{\lambda} \cos \theta_{x z}^{\lambda} \mathbf{k}$ is the illumination vector and $\mathbf{K}_{\mathbf{o}} \cong \mathbf{k}$ is the observation vector.

Using three wavelengths with three different lighting directions, we obtain a matrix relationship between the monochrome phase changes measured from the holograms and each component of the 3D displacement field.

$$
\left(\begin{array}{c}
\lambda_{1} \Delta \phi_{\lambda_{1}} \\
\lambda_{2} \Delta \phi_{\lambda_{2}} \\
\lambda_{3} \Delta \phi_{\lambda_{3}}
\end{array}\right)=2 \pi \mathbf{A}\left(\begin{array}{l}
u_{x} \\
u_{y} \\
u_{z}
\end{array}\right)
$$

The inversion of the matrix leads to the determination of the 3D displacement vector.

$$
\left(\begin{array}{l}
u_{x} \\
u_{y} \\
u_{z}
\end{array}\right)=\frac{1}{2 \pi} A^{-1}\left(\begin{array}{l}
\lambda_{1} \Delta \phi_{\lambda_{1}} \\
\lambda_{2} \Delta \phi_{\lambda_{2}} \\
\lambda_{3} \Delta \phi_{\lambda_{3}}
\end{array}\right)
$$

To simplify the notation, we note: $\theta_{R}=\theta_{x z}^{R}, \theta_{G}=\theta_{x z}^{G}$ et $\theta_{B}=\theta_{y z}^{B}$. In the setup, we have:

$$
\begin{aligned}
& K_{e}^{R}=\sin \theta_{R} i-\cos \theta_{R} k \\
& K_{e}^{G}=-\sin \theta_{G} i-\cos \theta_{G} k \\
& K_{e}^{B}=-\sin \theta_{B} i-\cos \theta_{B} k
\end{aligned}
$$

According to Eq. (5) the calculation of the three components of the displacements field is given by the following relation:

$$
\begin{aligned}
&\left(\begin{array}{l}
u_{x} \\
u_{y} \\
u_{z}
\end{array}\right)= \frac{1}{2 \pi \alpha} \\
&\left(\begin{array}{ccc}
1+\cos \left(\theta_{G}\right) & -1-\cos \left(\theta_{G}\right) & 0 \\
\sin \left(\theta_{G}\right)\left(1+\cos \left(\theta_{B}\right)\right) / \sin \left(\theta_{B}\right) & \sin \left(\theta_{R}\right)\left(1+\cos \left(\theta_{B}\right)\right) / \sin \left(\theta_{B}\right) & -\alpha / \sin \left(\theta_{R}\right) \\
-\sin \left(\theta_{G}\right) & -\sin \left(\theta_{R}\right) & 0
\end{array}\right) \\
& \times\left[\begin{array}{c}
\lambda_{R} \Delta \phi_{R} \\
\lambda_{G} \Delta \phi_{G} \\
\lambda_{B} \Delta \phi_{B}
\end{array}\right]
\end{aligned}
$$

where $\alpha=\sin \left(\theta_{R}\right)\left(1+\cos \left(\theta_{G}\right)\right)+\sin \left(\theta_{G}\right)\left(1+\cos \left(\theta_{R}\right)\right)$ and $\Delta \phi_{R}, \Delta \phi_{G}$, and $\Delta \phi_{B}$ are the optical phase changes between two deformation states, which are obtained from the reconstructed holograms, respectively, for the $\mathrm{R}, \mathrm{G}$, and $\mathrm{B}$ beams. In the setup, the angles are adjusted to $\theta_{R}=30.17, \theta_{G}=11.80$, and $\theta_{B}=46.27$. Figure $2 \mathrm{a}$ indicates the illuminating geometry and Fig. $2 \mathrm{~b}$ is a photograph illustrates the "white" illumination of the sample by the three laser wavelengths.

\section{Results and discussion}

The space specimens is glass/epoxy unidirectional laminates beam. The beam is $95 \mathrm{~mm}$ in length and $30 \mathrm{~mm}$ in width with a thickness of $4 \mathrm{~mm}$, is placed at distance $\mathrm{d}_{0}=830 \mathrm{~mm}$ from the color sensor. In other space

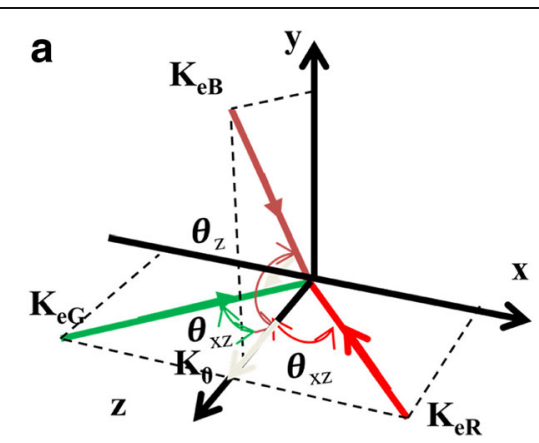

b

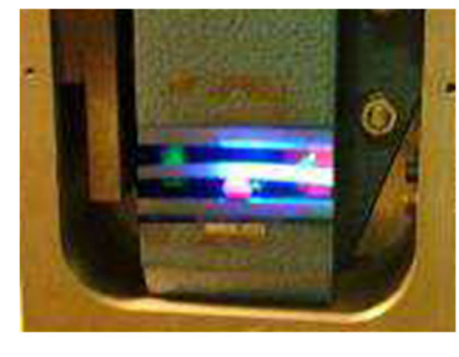

Fig. 2 a Illumination geometry, $\mathbf{b}$ photography of the mechanical head with a sample on test, white spot results of the mixing of the three R-G-B laser lines 


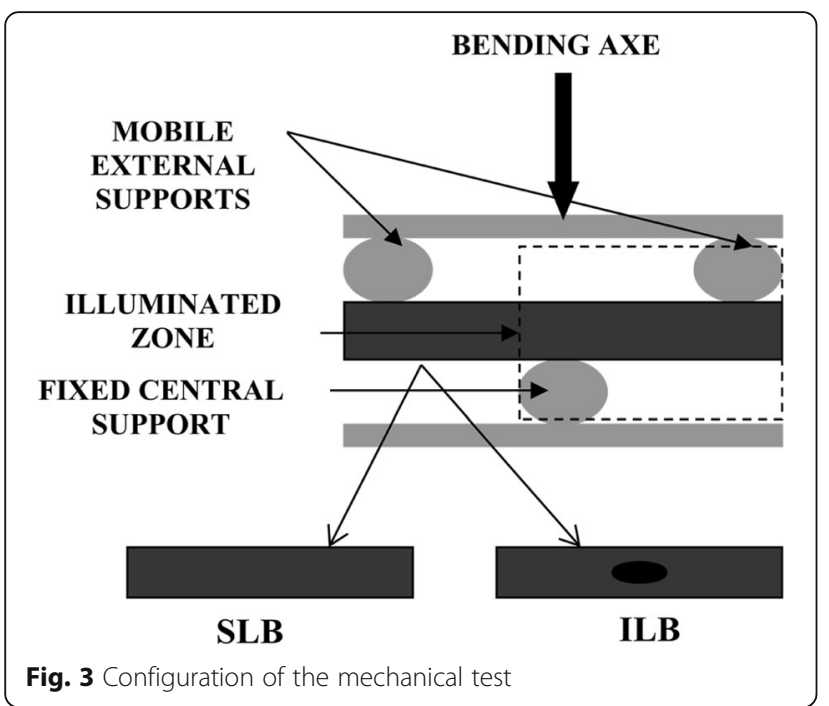

specimens we incorporate metal patch inside the laminate beam to see the influence of these patch on the behaviour of the laminates.

The Fig. 3 shows how the beam is submitted to a three point flexural loading.

During the test, the span supports are mobile and the central one is fixed. At each step of the test, the deformation is limited to a low fringe number in order to keep compatibility with the spatial resolution on the image of the sample. With each increment of displacement, the hologram of the current state is recorded and this hologram is used as a reference for the following state. The test runs until the fracture of the sample occurs.
During the test, we recorded 250 holograms for each specimen. The phase differences are calculated between two states of successive stresses. Figure 4 shows two sets of red, green and blue phase difference maps. Figure 4a corresponding to the phase difference between the hologram no. 1 and the reference initial hologram, numbered 0 . Figure $4 \mathrm{~b}$ corresponding to the phase difference between numbered 99 and numbered 98 .

We can note first for the series (a) the absence of the fringes for SLB since the constraint is weak at the beginning of the test. The fringes in ILB are related to a rigid body displacement.It is a first pitfall to avoid, the presence of fringes on the supports is useful to detect this problem. For series (b), more fringes were observed for both specimens. This means that the increment of deformation is higher for series (b) than for the series (a). For both series, the presence of noise between the fringes is observed for ILB, the experimental conditions are less good than for the SLB beam. A filtering of the fringes is therefore necessary.

To observe the influence of the patch, the whole of the phase maps were divided into 5 series and then these maps were unwrapped. Figure 5 shows the result obtained with ILB and SLB for series No. 3. The displacement maps are reset to compensate for the rigid body movements of the sample, which are inevitable at this measurement scale.

The background color corresponding to a zero displacement. The displacement maps computed for SLB are qualitatively validated by a MEF simulation of an embedded beam with a displacement imposed at $40 \mu \mathrm{m}$.

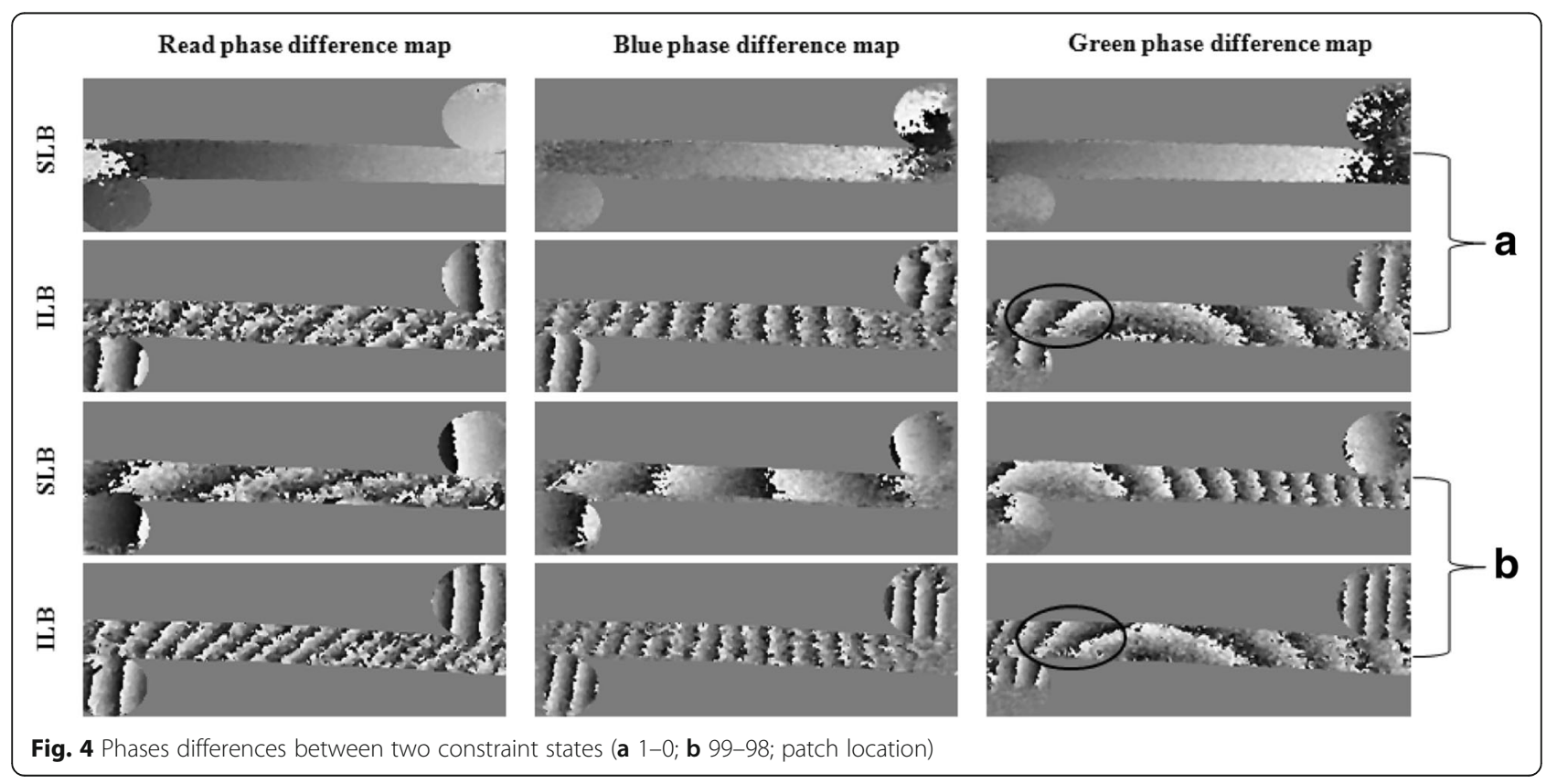




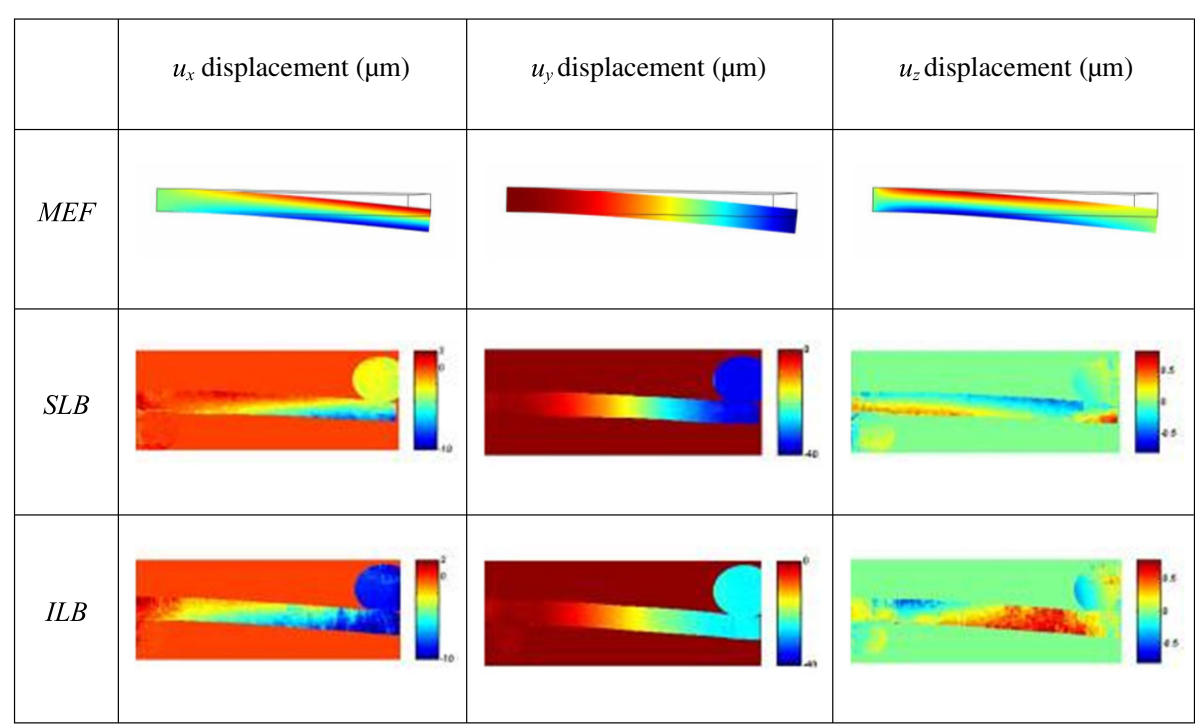

Fig. 5 The 3D displacements field (series $n^{\circ} 3$ )

The fine analysis of the components $u_{x}$ and $u_{y}$ of the displacement field reveals a difference in rigidity: the patch stiffens locally the sample. The component $u_{z}$ also reveals a Poisson effect on the quarter of the specimen which is due to the presence of the piezoelectric patch.

This result demonstrates in the one hand, that the sample, for the loading analyzed here, has not yet undergone compensation of the interlaminar delamination type.

In the other hand, the use of three primary colors (red, green and blue for example) digital holography led to the absolute measurement of the displacement field or index variation [17], which was not the case in monochrome holography [18-20].

However, these applications are based on the use of a monochromatic source that greatly considerably complicates the experimental set-up (three different reference waves) and leads to a loss of spatial resolution [21].

With the advent of color cameras such as the CMOS sensor used, it is now possible to simultaneously record colors on the same sensor. The reference waves then have the same incidence and the segmentation of the colors is carried out by the sensor.Thus the experimental set-up becomes very simple and the spatial resolution is maximum because the orders share the same frequency space.

Finally, the reconstruction of the color holograms can be carried out independently for each wavelength by convolution, provided that the size of the monochromatic holograms remains identical for a perfect superposition to the ready pixel [22].

\section{Conclusion}

In conclusion, this Letter presents a three-color digital holographic method for real-time 3D deformation measurements. The setup is considerably simplified, because a unique reference beam can be used to simultaneously record the three-color digital holograms. Thanks to the reconstruction of each individual monochrome hologram using a convolution algorithm, the three images are superposed. The application of the method to real-time 3D measurement shows satisfactory results compared with a pure sequential monochrome measurement, which validates the concept. This paper demonstrates that three dimensional measurements make it possible to identify on the visible edge of the sample the displacements fields. In addition from this analysis we have shown the effect of the embedded pastilles which it stiffens locally the sample. Also we remark a Poisson effect on the quarter of the specimen but, has not yet undergone compensation of the interlaminar delamination type.

Consequently, three-color digital holographic method is a very efficient technique used in non-destructive testing and characterizing of composites, since many kinds of defects may be simultaneously detected over large areas, without any contact with the tested material.

\section{Abbreviations}

B: Blue line; $d_{r}$ : Reconstruction distance; G: Green line; ILB: Incorporated laminate beam; M: Mirror; MEF: Model element fini; PBS: Polarizer beam splitter; R: Red line; $R_{c}$ : Curvature radius; SLB: Simple laminate beam;

$\theta_{B}$ : Angle blue; $\theta_{G}$ : Angle green; $\theta_{R}$ : Angle red

\section{Acknowledgments}

The authors are grateful to IUT members in Mans for his help in preparing the material studied.

\section{Funding}

The financial support of LAUM UMR CNRS of Mans (France) and scientific research of Tunisia is acknowledged.

Availability of data and materials Not applicable. 


\section{Authors' contributions}

C Poilane, P Picart and M Gargouri conceived the project. C Poilane, P Picart and M Karray designed and performed the experiments. C Poilane, P Picart and M Karray analyzed the data. M Karray wrote the manuscript. All the authors discussed the results and commented on the manuscript at all stages. All authors read and approved the final manuscript.

Ethics approval and consent to participate

Not applicable.

Consent for publication

Not applicable.

\section{Competing interests}

The authors declare no competing financial interests.

\section{Publisher's Note}

Springer Nature remains neutral with regard to jurisdictional claims in published maps and institutional affiliations.

\section{Author details}

'Unité de l'état solide, Faculté des Sciences de Sfax, Route Soukra, 3018 Sfax, Tunisie. ${ }^{2}$ LAUM UMR CNRS 6613, Université du Maine, Av. O. Messiaen, 72085 Le Mans, France. ${ }^{3}$ CIMAP UMR6252, Université de Caen, 6 Boulevard du Maréchal Juin, 14050 Caen cedex 4, France.

Received: 24 April 2017 Accepted: 20 September 2017

Published online: 29 September 2017

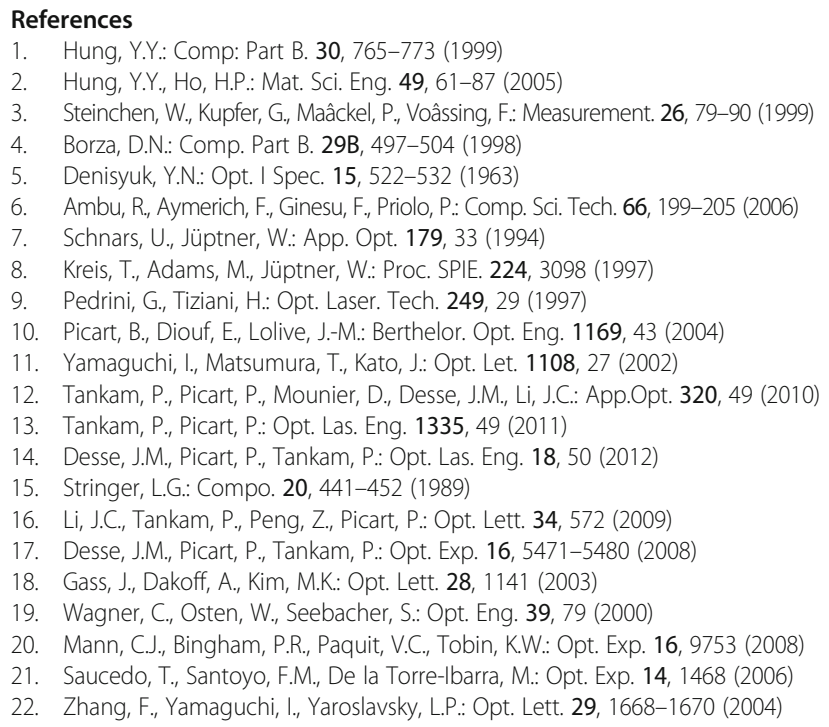

\section{Submit your manuscript to a SpringerOpen ${ }^{\mathcal{O}}$ journal and benefit from:}

- Convenient online submission

- Rigorous peer review

- Open access: articles freely available online

- High visibility within the field

- Retaining the copyright to your article 